\title{
sciendo
}

\section{Analysis of Goal Scoring Patterns in the 2018 FIFA World Cup}

\author{
by \\ Alliance Kubayi ${ }^{1}$
}

The aim of this study was to analyse the goal scoring patterns during the 2018 FIFA World Cup. All goals scored during the tournament were analysed using the InStat video analysis system. The results showed that 169 goals (open play: $60.9 \%$; set play: $39.1 \%$ ) were scored during the competition. While 85 goals (82.5\%) were scored from elaborate attacks, 18 goals (17.5\%) came from counter attacks. A chi-square test indicated that there was a significant difference in the type of possession $(\chi 2(1, n=103)=43.58, p=0.00)$. The highest number of goals was yielded from the final third $(35 \%)$ as compared to the first (33\%) and middle (32\%) thirds. The results also indicated that most goals accounted from short passes $(69.9 \%)$, while $13.6 \%$ of goals came from long passes and $16.5 \%$ from mixed passes $(\chi 2(2, n=103)=62.12$, $p=0.00)$. Soccer coaches should incorporate set pieces in their training sessions in view to improve goal-scoring opportunities.

Key words: goals, set pieces, open play, tactics, performance.

\section{Introduction}

Soccer is the world's favourite sport, played by over 240 million players in 1.4 million teams registered in 300,000 clubs around the world (Acar et al., 2009). Every four years, billions of people in more than 200 countries watch the month-long FIFA World Cup, which is undoubtedly the biggest tournament in association soccer (Acar et al., 2009). The World Cup is the greatest prize in soccer and provides an opportunity to examine the best national teams and players in the world. In addition to the anthropometric, psychological and physiological research, analysis of performance of soccer players in the field in terms of techniques and tactics has become popular (Acar et al., 2009).

Scoring goals is the ultimate determinant of successful soccer team performance and has received extensive attention among researchers (Jones et al., 2004; Tenga et al., 2010; Yiannakos and Armatas, 2006). This is prompted by the fact that goal scoring is paramount for any team's success (Hughes and Churchill, 2005). Armatas et al. (2009) evaluated goals scored in soccer matches during the 2006-2007 Greek Super League season and found that $59 \%$ of the goals were scored in the second half. Alberti et al. (2013) also indicated that a significantly higher frequency of goals was recorded in the second half, with the highest scoring rate noted in the final 15-min period of the game during major European soccer leagues.

Mitrotasios and Armatas (2014) analysed 76 goals scored from 31 matches during the 2012 European Football Championship in PolandUkraine. They reported that most goals were scored during open play $(72.4 \%)$, while $27.6 \%$ came from set play. The possession style of play and corner kicks were the most productive sources of open play and set piece goals, respectively. Regarding the starting zone of attack, $56.6 \%$ of goals began from the attacking half, with $43.4 \%$ from the defending half. This demonstrates that teams should tailor their tactics in such a way that they win ball possession in the final third in order to improve goal scoring opportunities. With regard to the different lengths of possession, $61.8 \%$ of goals were scored from less than four passes (Mitrotasios and Armatas, 2014).

Despite that a substantial body of literature

1 - Department of Sport, Rehabilitation and Dental Sciences, Tshwane University of Technology, South Africa.

Authors submitted their contribution to the article to the editorial board.

Accepted for printing in the Journal of Human Kinetics vol. 71/2020 in January 2020. 
exists on goal scoring patterns in soccer (Acar et al., 2009; Mitrotasios and Armatas, 2014; Yiannakos and Armatas, 2006), there is a need to analyse how and when goals are scored in soccer because the game tactics continuously evolve and it is important for coaches to be cognisant of recent trends in goal scoring patterns especially at the FIFA World Cup. Additionally, such information could be very useful to soccer coaches in designing and implementing training programmes and choosing appropriate goal scoring tactics, thus enhancing the chances of team success (Yiannakos and Armatas, 2006). Therefore, this study was carried out to analyse the goal scoring patterns during the 2018 FIFA World Cup.

\section{Methods}

\section{Match sample and data source}

A total of 169 goals were scored at the group and knockout stages of the FIFA World Cup held in Russia from the $14^{\text {th }}$ of June to the $15^{\text {th }}$ of July 2018. Videotapes of all goals scored during the tournament were analysed using the InStat video analysis system. InStat is a Russian-based private company that analyses individual and team performances in soccer. Ethical clearance was obtained from the Ethics Committee of Tshwane University of Technology, Pretoria, South Africa.

\section{Performance variables}

Performance variables in the present study consisted of (1) the number of goals scored from set pieces (corner kicks, free kicks, penalties, throwins), (2) the frequency of goal scoring from open play during a 15 -min interval $(1-15,16-30,31$-half time, 45-60, 61-75, 76-full time), and (3) the offensive variables of goals scored from open play. Offensive variables included the team possession type (counter and elaborate attacks), starting zone (first, middle and final thirds), pass number (short, medium and long possession), and pass length (short, long and mixed passes). Operational definitions of these offensive variables are provided in Table 1.

\section{Reliability testing}

An intra-observer test using the kappa measure of agreement was undertaken to assess the reliability of all variables. Of 103 goals scored from open play, $13(12.6 \%)$ randomly selected were analysed and reanalysed by the principal investigator after three weeks in order to reduce learning effects (O'Donoghue, 2015). The kappa values ranged from 0.81 to 1.00 , demonstrating that the strength of agreement was very good for all performance variables (Table 2).

\section{Statistical analysis}

Descriptive statistics such as frequencies and percentages were used to analyse the data. A chi-square goodness-of-fit test was applied to examine whether any significant differences existed regarding the possession type, starting zone, pass number and pass length. A probability level of $p \leq 0.05$ was used to indicate significance. Data were analysed using the Statistical Package for Social Sciences (SPSS) version 25.

\section{Results}

The results showed that 169 goals were scored with an average of 2.64 goals per match during the tournament. While 66 goals $(39.1 \%)$ were scored from set play, 103 (60.9\%) came from open play. From a set play perspective, most goals emerged from penalties (34.9\%), followed by corner kicks (31.8\%), free kicks (30.3\%) and throwins $(3.0 \%)$. Concerning the frequency of goal scoring during open play, most goals were scored in the second half $(63 \%)$, with the highest number of goals recorded in the 45-60 minutes of the game (28\%) (Figure 1). Presented in Table 2 are summary statistics of goals scored from open play.

Eighty-five goals $(82.5 \%)$ came from elaborate attacks, while 18 goals $(17.5 \%)$ resulted from counter attacks. A chi-square test showed that there was a significant difference in the type of possession $\left(\chi^{2}(1, \mathrm{n}=103)=43.58, p=0.00\right)$. Short ball possession $(50.5 \%)$ yielded a significantly higher number of goals scored than long (31.1\%) and medium (18.4\%) ball possession. With regard to the starting zone, 35,33 and $32 \%$ goals resulted from the final, first and middle thirds, respectively $(p>0.05)$. The results also indicated that most goals came from short passes $(69.9 \%)$ in contrast to long $(13.6 \%)$ and mixed (16.5\%) passes, which yielded a significant difference $(\chi 2(2, \mathrm{n}=103)=62.12, p=$ $0.00)$. 
Table 1

Operational definitions of offensive variables (Tenga et al., 2010)

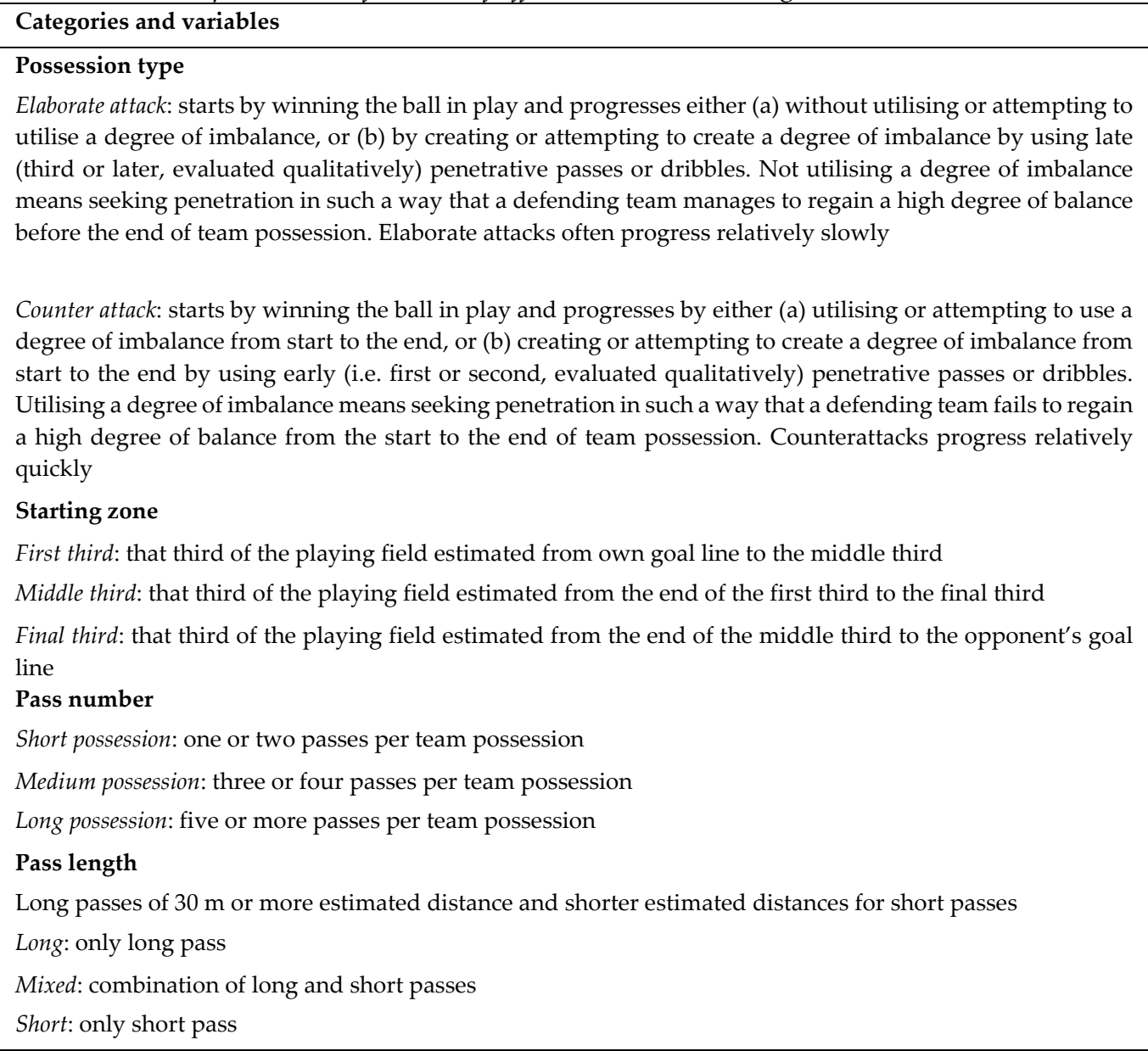

Table 2

Reliability test analysis

\begin{tabular}{llll}
\hline Reliability test & Variable & Kappa value & $\begin{array}{l}\text { Strength of Agreement } \\
\text { (Altman, 1995) }\end{array}$ \\
\hline Intra-observer & Possession type & 0.81 & Very good \\
Intra-observer & Starting zone & 0.88 & Very good \\
Intra-observer & Pass number & 0.88 & Very good \\
Intra-observer & Pass length & 0.86 & Very good \\
Intra-observer & Frequency of goal scoring & 1.00 & Very good \\
\hline
\end{tabular}




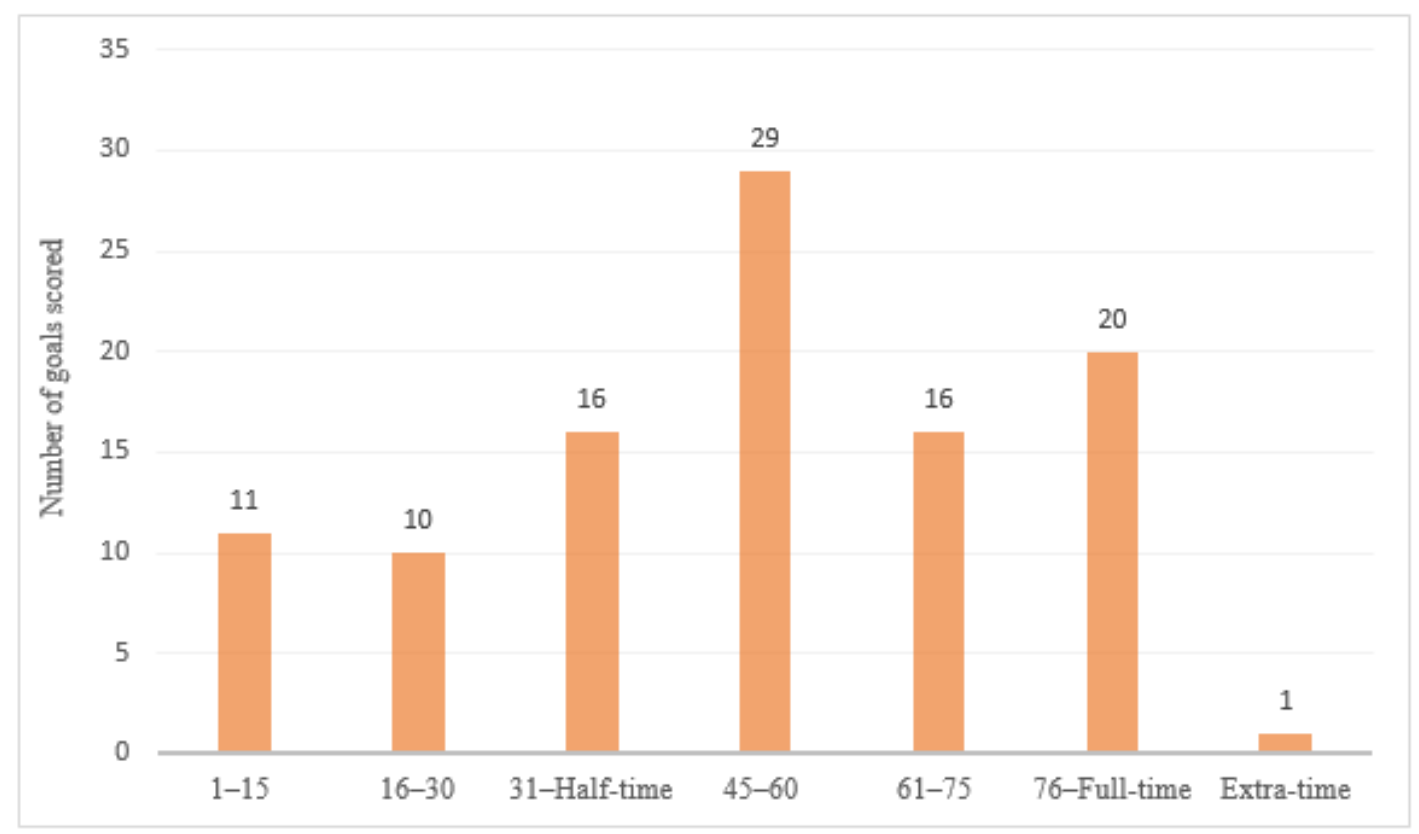

Figure 1

Number of goals scored per 15-min period

Table 3

Offensive variables of goals scored from open play during the tournament

\begin{tabular}{llccc}
\hline & $\mathrm{n}$ & $\%$ & $\chi^{2}$ & $p$ \\
\hline Possession type & & & 43.58 & $0.00^{*}$ \\
Elaborate attack & 85 & 82.5 & & \\
Counter attack & 18 & 17.5 & 0.14 & \\
Starting zone & & & & \\
First third & 34 & 33.0 & $0.00^{*}$ \\
Middle third & 33 & 32.0 & \\
Final third & 36 & 35.0 & & \\
Pass number & & & & \\
Short possession & 52 & 50.5 & & \\
Medium possession & 19 & 18.4 & & \\
Long possession & 32 & 31.1 & & \\
Pass length & & & & \\
Short pass & 72 & 69.9 & & \\
Long pass & 14 & 13.6 & & \\
Mixed pass & 17 & 16.5 & & \\
\hline
\end{tabular}

*Significant at 0.05 


\section{Discussion}

The purpose of this study was to investigate the goal scoring patterns at the 2018 FIFA World Cup. The findings show that $63 \%$ of goals were recorded during the second half of the game. This scoring rate was higher than $57 \%$ of goals scored in the second half during the 2014 FIFA World Cup in Brazil (Michailidis, 2014). The high number of goals scored during the second half could be explained by the fact that players' physical performance tends to deteriorate during this period due to the onset of fatigue. Consequently, fatigued players experience lapses in concentration that may lead to tactical errors, thereby increasing the vulnerability of the defence to conceding goals (Carling et al., 2005).

The results of this study further showed that most goals were scored in the first 15 minutes of the second half and the latter part of the game. Specifically, players seek physical means of registering their presence on the opposition at the start of the second half, whilst in the last quarter such players contest for possession and usually become more desperate. Therefore, the late surge in goals scored may be an inherent factor in the game as play becomes more exigent towards the end (Carling et al., 2005). Teams that are losing seem to have a sense of urgency towards the end of the game because they always strive to draw or win during tournaments. Subsequently, such teams usually take risks by pushing more players forward to gain an advantage over the opponents in order to create more goal scoring chances, thereby scoring or conceding goals (Reilly, 1997).

From a set play viewpoint, the highest number of goals at the 2018 FIFA World Cup emerged from penalties. This could be attributed to the use of Video Assistant Referees (VARs) which was designed to review video footage on contested or uncertain decisions and advise the referee accordingly if a wrong call was made (FIFA, 2018). Consequently, more penalties were awarded which resulted in most goals being scored from this type of a set piece. The finding in which most goals were scored from corner kicks substantiates the results reported by Njororai (2013), who indicated that corner kicks were the most productive sources of set piece goals in the 2010 FIFA World Cup competition hosted in South Africa. Relatively few goals scored through throw-ins in this study could be attributed to the fact that they provide an excellent opportunity to maintain ball possession (Carling et al., 2005). Overall, the upward trend in the number of goals scored from set plays was observed, increasing from $22 \%$ in the 2014 World Cup to $39 \%$ in the 2018 World Cup. This result demonstrates that scoring from open play is becoming more difficult and consequently, soccer coaches should pay more attention to practicing set plays in their training sessions in order to create more goal-scoring opportunities.

Additionally, more goals resulted more from short passes than long passes. Oberstone (2009) reiterated that employing short passes was a more reliable way of advancing the ball to the opponent's half than moving the ball with a higher proportion of less accurate, long passes. The finding in which most goals came from short possession is consistent with that of Wright et al. (2011). Acar et al. (2009) are of the view that playing fast and being well organised in attacking positions yields more scoring opportunities. The current study indicated that a higher number of goals emanated from the final third than the first and middle thirds of the field. However, it should be noted that regaining ball possession in the final third requires players to have high levels of fitness needed to efficiently execute match tactics which involves pressurising the defence throughout the game (Wright et al., 2011).

\section{Practical implications}

The findings of the present study provide practical implications for coaches. Soccer scientists and coaches should modify their training programmes such that players are wellconditioned in order to tolerate fatigue. This will ensure that players have high levels of fitness to maintain concentration and match tactics efficiently throughout the game. Soccer coaches should pay more attention to practicing set plays, especially corner kicks and free kicks, more effectively because of their potential to team success. Finally, coaches should deploy tactics which would allow players to play short passing game and regain ball possession in the opponent's half in order to increase goal-scoring opportunities.

\section{Conclusion}

The results indicated that most goals were scored during the second half of the game, with a greater number of goals conceded in the first 15 minutes of the second half and the final period of 
the game. While a downward trend was observed in goals scored during open play, there has been an increase in the number of goals scored from set plays in the last two successive world cups. More goals were scored from short passes than long and mixed passes. Most goals emerged from the final third in contrast to first and middle thirds.

\section{References}

Acar MF, Yapicioglu B, Arikan N, Yalcin S, Ates N, Ergun, M. Analysis of goals scored in the 2006 world cup. In T Reilly, F Korkusuz (Eds.), Proceedings of the sixth world congress on science and football, science and football VI (pp. 233-242). London: Routledge; 2009

Alberti G, Iala FM, Arcelli E, Cavaggioni L, Rampinini E. Goal scoring patterns in major European soccer leagues. Sports Sci Health, 2013; 9: 151-153

Armatas V, Yiannakos A, Zaggelidis G, Papadopoulou S, Fragkos N. Goal scoring patterns in Greek top leveled soccer matches. J Phys Educ Sport, 2009; 23: 1-5

Carling C, Williams A, Reilly T. The handbook of soccer match analysis: A systematic approach to improving performance. London: Routledge; 2005

FIFA. How does video assistant referee improve the game? Available at www.FIFA.com/VARat; accessed on 01.10.2018

Hughes MD, Churchill S. Attacking profiles of successful and unsuccessful team in Copa America 2001. In: Science and Football V. Eds: Reilly T, Cabri J, Araújo D (Eds), London and New York: Routledge; 219-224; 2005

Jones P, James N, Mellalieu S. Possession as a performance indicator in soccer. Int J Perform Anal Sport, 2004; 4: 98-102

Michailidis, Y. Analysis of goals scored in the 2014 World Cup soccer tournament held in Brazil. Int J Sport Stud, 2014; 4(9): 1017-1026

Mitrotasios M, Armatas V. Analysis of Goal Scoring Patterns in the 2012 European Football Championship. The Sport Journal, 2014. Available at http://thesportjournal.org/article/analysis-of-goal-scoringpatterns-in-the-2012-european-football-championship; accessed on 05.10.2018

Oberstone J. Differentiating the top English Premier League football clubs from the rest of the pack: Identifying the keys to success. J Quant Anal Sport, 2009; 5: 10

O'Donoghue P. An introduction to performance analysis of sport. Abingdon: Routledge; 2015

Njororai WWS. Analysis of goals scored in the 2010 world cup soccer tournament held in South Africa. J Phys Educ Sport, 2013; 13: 6-13

Reilly T. Energetics of high intensity exercise (soccer) with particular reference to fatigue. J Sports Sci, 1997; 15(3): 257-263

Tenga A, Holme I, Ronglan LT, Bahr R. Effect of playing tactics on achieving score-box possessions in a random series of team possessions from Norwegian professional soccer matches. J Sports Sci, 2010; 28(3): 245-255

Yiannakos A, Armatas V. Evaluation of the goal scoring patterns in European Championship in Portugal 2004. Int J Perform Anal Sport, 2006; 6(1): 178-188

Wright C, Atkins S, Polman R, Jones B, Sargeson L. Factors associated with goals and goal scoring opportunities in professional soccer. Int J Perform Anal Sport, 2011; 11(3): 438-449

\section{Corresponding author:}

\section{Alliance Kubayi}

Tshwane University of Technology

Department of Sport, Rehabilitation and Dental Sciences, Republic of South Africa; Pretoria; 0001

Telephone number: +27 12382 4272; Fax number: +27 12382 5801; Email address: kubayina@tut.ac.za 